\title{
MAXIMAL IDEAL TRANSFORMS OF NOETHERIAN RINGS
}

\author{
JACOB R. MATIJEVIC
}

\begin{abstract}
Let $R$ be a commutative Noetherian ring with unit. Let $T$ be the set of all elements of the total quotient ring of $R$ whose conductor to $R$ contains a power of a finite product of maximal ideals of $R$. If $A$ is any ring such that $R \subset A \subset T$, then $A / x A$ is a finite $R$ module for any non-zerodivisor $x$ in $R$. It follows that if, in addition, $R$ has no nonzero nilpotent elements, then any ring $A$ such that $R \subset A \subset T$ is Noetherian.
\end{abstract}

Let $R$ be a commutative Noetherian domain with unit of Krull dimension one. The Krull-Akizuki theorem [6, Theorem 33.2] states that if $T$ is an integral domain containing $R$ and contained in a finite algebraic extension of the quotient field of $R$ then $T$ is Noetherian. By adjoining a finite number of elements to $R$ and letting this new ring be called $R$, one proves the theorem by proving the following: Any ring $A$ between a Noetherian domain $R$ of Krull dimension one and its quotient field is Noetherian. This is equivalent to showing that if $x$ is any nonzero element of $R$ then $A / x A$ is a finite $R$ module.

We shall restate this reduction of the Krull-Akizuki theorem in such a way that the final statement is true for any Noetherian ring of any Krull dimension. In order to do this we first characterize the relationship between a one dimensional Noetherian domain $R$ and its quotient field $T$. If $y$ belongs to $T$, then its conductor to $R$, or its denominator ideal contains some powered product of a finite number of maximal ideals of $R$. In the special case that $R$ has precisely one maximal ideal $M, T$ is the set of all elements whose conductor to $R$ contains a power of $M$.

If $B$ is any commutative Noetherian ring with unit and $I$ is any ideal of $B$ that contains a non-zero-divisor, the $I$-transform of $B$ is defined to be the set of all elements of the total quotient ring whose conductor to $B$ contains a power of $I$. The $I$-transform is a ring between $B$ and its total quotient ring. If $B$ is a Noetherian domain of Krull dimension one and $B$ has one maximal ideal $M$, then the quotient field is the $M$-transform of $B$.

Now if $R$ is any commutative Noetherian ring with unit, we call $T$ the global transform of $R$ if $T$ is the set of all elements of the total quotient ring whose conductor to $R$ contains a power of a finite product of maximal ideals of $R$. If $M$ is any maximal ideal of $R, T_{M}=T \otimes_{R} R_{M}$, where $R_{M}$ is the localization of $R$ at $M$, is the $M R_{M}$-transform of $R_{M}$. Also $T$ contains the $M$-transform of $R$ for $M$ any maximal ideal of $R$. If $R$ is a domain of Krull dimension one, we

Received by the editors May 16, 1974 and, in revised form, November 4, 1974.

AMS (MOS) subject classifications (1970). Primary 13E05, 13E10; Secondary 13 B99.

Key words and phrases. Conductor, ideal transform, global transform, Krull dimension, KrullAkizuki theorem, Noetherian ring, Artinian module. 
have already remarked that $T$ is the quotient field of $R$. The global transform proves to be the appropriate generalization of the quotient field in the reduction of the Krull-Akizuki theorem that makes the conclusion true for rings of arbitrary Krull dimension.

In the following, all rings are commutative with unit, and all modules are unitary. By the term "finite $R$ module" we mean finitely generated $R$ module. A local ring is a Noetherian ring with a unique maximal ideal. The basic reference for the notation and terminology is [3].

THEOREM. If $R$ is a Noetherian ring and $T$ is the global transform of $R$, then for any ring $A$ such that $R \subset A \subset T, A / x A$ is a finite $R$ module for each nonzero-divisor $x$ in $R$.

The following proof incorporates a simplification, due to W. Schelter, to part of the author's original proof.

Proof. We will attempt to show that $A \subset R x^{-n}+x A$ for some $n>0$. This will suffice for the result. To do this we first show that for any $a$ in $A$ there exists a $k>0$ such that $a \in R x^{-k}+x A$. Let $J=\{r \in R \mid r a \in R\}$. By definition of $T, J$ contains a finite product of maximal ideals of $R$. Hence $R / J$ is an Artinian ring. The images of the ideals $\left\{\left(x^{m}\right)\right\}_{m>0}$ stabilize in $R / J$. Hence for some $k,\left(x^{k}\right)+J=\left(x^{k+1}\right)+J$. We can then write $x^{k}=r x^{k+1}+j$ for some $r \in R, j \in J$. But then $a x^{k}=a r x^{k+l}+a j$ or $a=a r x+a j x^{-k} \in x A$ $+R x^{-k}$ since $a j \in R$. To establish the result we begin by noting that the descending chain of ideals $I_{h}=\left(x^{h} A \cap R, x R\right)$ stabilizes for some $h=n$. This is the case since the images of these ideals stabilize in $(x A \cap R) / x R$, which is a module of finite length. (It is a finite $R$ module, generated by images of elements of the form $x a_{i}, 1 \leqslant i \leqslant w$, with $a_{i} \in A$. There is a finite product of maximal ideals, call it $I$, such that $I a_{i} \subset R$ for each $i$. Hence $(x A \cap R) / x R$ is a finite $R / I$ module.) If there is an $a \in A$ such that $a \notin R x^{-n}+x A$, then choose $m>n$ minimal such that $a \in R x^{-m}+x A$. Hence $a=r x^{-m}+x a^{\prime}$, or $x^{m} a=r+x^{m+1} a^{\prime}, a^{\prime} \in A, r \in R . x^{m}\left(a-x a^{\prime}\right)=r \in I_{m}=I_{m+1}$. Hence $x^{m}\left(a-x a^{\prime}\right)=x^{m+1} a^{\prime \prime}+x r^{\prime}, a^{\prime \prime} \in A, r^{\prime} \in R$. Rearranging, $a=x\left(a^{\prime \prime}+a^{\prime}\right)$ $+r^{\prime} x^{-(m-1)} \in R x^{-(m-1)}+x A$, a contradiction.

The key to the above proof was showing that the module $(x A \cap R) / x A$ was an Artinian module. In the proof of the Krull-Akizuki theorem [3, Theorem 93], the above module, being a submodule of $R / x R$, is readily seen to be Artinian, since $R$ has Krull dimension one.

We note an immediate Corollary.

COROLlaRY. Let $R$ be a Noetherian ring with no nonzero nilpotent elements and $T$ the global transform of $R$. Then any ring $A$ such that $R \subset A \subset T$ is Noetherian.

Proof. Let $Q$ be a minimal prime ideal of $A \cdot Q \cap R=P$ is a minimal prime ideal of $R . A / Q$ is contained in the global transform of $R / P$. Since $R / P$ is a domain, $A / Q$ is Noetherian by the Theorem. It follows that $A$ is Noetherian.

Some special cases of these results were already known. Ferrand and Raynaud [1] showed in the context of the above Corollary that $T$ was 
Noetherian if $T$ was integral over $R$. Flexor-Mangeney [2] showed that if $R$ was a local domain with maximal ideal $M$ and the integral closure of $R$ had one maximal ideal, then, if $T$ is the $M$-transform of $R, T / x T$ is a finite $R$ module for each nonzero $x$ in $R$. She was also the first to notice the "KrullAkizuki" behavior between the rings $R$ and $T$.

A natural question to ask in light of the above Corollary is: Can the global transform $T$ of a Noetherian ring $R$ be Noetherian if $R$ is allowed to have nonzero nilpotent elements? Ferrand and Raynaud give an example [1, Proposition 3.3] of a local domain $R$ of Krull dimension two such that $T$, the maximal ideal transform of $R$, is integral over $R$, yet $T$ is not a finite $R$ module. If $\hat{R}$ is the completion of $R$ with maximal ideal $\hat{M}$, then the $\hat{M}$-transform of $\hat{R}$ is $T \otimes_{R} \hat{R}=T^{\prime}$, and $T^{\prime}$, is integral over $\hat{R}$. If $T^{\prime}$ were Noetherian, then, since $T^{\prime} / \hat{M} T^{\prime}$ is a finite $\hat{R}$ module, $T^{\prime}$ would be a finite $\hat{R}$ module [6, Theorem 30.6]. But then $T$ must itself be a finite $R$ module, a contradiction.

If $T$ is the maximal ideal transform of a local domain $R, T$ need not be integral over $R$. Although this is clear in case $R$ has Krull dimension one, examples in higher dimension are not as easy to find. Ferrand and Raynaud [1] give conditions on the ring $R$ so that $T$ will be integral over $R$. Examples when $T$ is not integral over $R$ are given by the rings $R$ of Nagata's counterexample to the saturated chain condition [6, Example 2, when $R$ is of any Krull dimension] or [4, pp. 23-27, when $R$ has Krull dimension two]. In that example, $R$ is a local domain of Krull dimension $n$ whose integral closure $R^{\prime}=V \cap W$, where $V$ is a rank one discrete valuation ring and $W$ is a regular local ring of Krull dimension $n$. The maximal ideal transform $T$ of $R$ in this case is $T=W$, which properly contains $R^{\prime}$.

There is no immediate extension of the Theorem to ideal transforms of a Noetherian ring at ideals other than maximal ones. Nagata [5] gives an example of an ideal transform at a rank one prime ideal of a local domain of Krull dimension two, which is not Noetherian.

Even if the ideal transform $T$ at a nonmaximal prime ideal $P$ of a local domain $R$ is Noetherian, it does not follow for any ring $A$ such that $R \subset A \subset T$ that $A$ need be Noetherian. As an example let $R=k[X, Y]_{(X, Y)}$ where $X, Y$ are indeterminates, $k$ any field. If $P=(X)$ then $T=R_{S}$ where $S=\left\{1, X, X^{2}, \ldots\right\}$. If

$$
A=k\left[X, Y / X, Y / X^{2}, \ldots, Y / X^{n}, \ldots\right]_{N}
$$

where $N=(X, Y / X, \ldots)$ then $R \subset A \subset T . A$ is not Noetherian, since if $J=\bigcap_{n>0} X^{n} A$, then $0 \neq Y \in J$.

For a study of pairs of Noetherian rings $R \subset T$ for which every intermediate ring is Noetherian ( $R, T$ as in the Corollary) we refer the reader to [7].

\section{BIBLIOGRAPHY}

1. D. Ferrand and M. Raynaud, Fibres formelles d'un anneau local noethérien, Ann. Sci. École Norm. Sup. (4) 3 (1970), 295-311. MR 42 \#7660.

2. M. Flexor-Mangeney, Une proprieté des anneaux unibranches, Bull. Sci. Math. 96 (1972), $169-175$.

3. I. Kaplansky, Commutative rings, Allyn and Bacon, Boston, Mass., 1970. MR 40 \#234.

4. - Topics in commutative rings. I (mimeographed notes). 
5. M. Nagata, Lectures on the fourteenth problem of Hilbert, Tata Inst. Fund. Res. Lectures on Math., no. 31, Tata Institute of Fundamental Research, Bombay, 1965. MR 35\#6663.

6 , Local rings, Interscience Tracts in Pure and Appl. Math., no. 13, Interscience, New York, 1962. MR 27 \#5790.

7. A. Wadsworth, Pairs of domains where all intermediate domains are Noetherian, Trans. Amer. Math. Soc. 195 (1974), 201-211.

8. W. Schelter, On the Krull-Akizuki theorem (preprint).

Department of Mathematics, University of Kentucky, Lexington, Kentucky 40506

Current address: Department of Mathematics, University of Southern California, Los Angeles, California 90007 